\title{
Heavy-Quark Form Factors and Threshold Cross Section at $O\left(\alpha_{S}^{2}\right)$
}

Roberto Bonciani* ${ }^{\dagger}$

Dep. de Física Teòrica, IFIC - CSIC, Universitat de València, E-46071 Valencia, Spain

E-mail: Roberto.Boncianilific.uv.es

\section{Werner Bernreuther}

Inst. für Theoretische Physik, RWTH Aachen, D-52056 Aachen, Germany

E-mail: breuther@physik.rwth-aachen.de

\section{Thomas Gehrmann}

Inst. für Theoretische Physik, Universität Zürich, CH-8057 Zürich, Switzerland

E-mail: gehrtephysik.unizh.ch

\section{Roland Heinesch}

Inst. für Theoretische Physik, RWTH Aachen, D-52056 Aachen, Germany

E-mail: heinesch@physik.rwth-aachen.de

\section{Thilo Leineweber}

Inst. für Theoretische Physik, RWTH Aachen, D-52056 Aachen, Germany

E-mail: leineweberaphysik.rwth-aachen.de

\section{Pierpaolo Mastrolia}

Dep. of Physics and Astronomy, UCLA, Los Angeles, CA 90095-1547

E-mail: mastrolia@physics.ucla.edu

\section{Ettore Remiddi}

Dip. di Fisica, Università di Bologna, and INFN, Sezione di Bologna, I-40126 Bologna, Italy

E-mail: Ettore.Remiddi@bo.infn.it

During the last year, analytic expressions for the two-loop QCD corrections to the form factors for the vector, axial-vector, scalar and pseudo-scalar vertices involving a pair of heavy quarks, $Q \bar{Q}$, were calculated. The results are valid for arbitrary momentum transfer and mass of the heavy quarks. These form factors have a number of applications, including anomalous couplings, the $e^{+} e^{-} \rightarrow Q \bar{Q}$ cross section, and the forward-backward asymmetry of heavy quarks. Here the $Q \bar{Q}$ threshold cross section is presented with some new second order axial vector contributions.

International Europhysics Conference on High Energy Physics

July 21st - 27th 2005

Lisboa, Portugal

\footnotetext{
${ }^{*}$ Speaker.

†Work supported by the European Union under the contract HPRN-CT2002-00311 (EURIDICE)
} 
In the next years particle physics will receive a big boost, due mainly to the start of activity of the Large Hadron Collider (LHC) that will explore physics at the TeV scale. One of the purposes of such a program is the understanding of the electroweak symmetry breaking and in particular a possible confirmation of the Higgs mechanism, via the discovery of the Higgs boson, the last still missing particle of the Standard Model (SM). In this context, an important role will be played by heavy quarks. This is the sector of the SM where a possible deviation from the Higgs mechanism could be detected first (in particular for the top quark that has a large mass and thus a large coupling to the Higgs). Therefore, a precise theoretical determination of observables concerning the $b$ - and $t$-quark production and decay processes is mandatory in order to match the precision that will be required by the Tevatron, LHC and by an International Linear Collider.

A step in this direction was made in the last year with the calculation of the QCD two-loop corrections to the form factors for the production of heavy quarks in $e^{+} e^{-}$collisions. In [1], the vector current was considered (see also [2]). Using the Laporta algorithm [3] for the reduction of the dimensionally-regularized scalar integrals to the set of master integrals and the differential equations technique [4] for their calculation [5], the $O\left(\alpha_{S}^{2}\right)$ corrections to the form factors for the vertex $\gamma Q \bar{Q}$ were evaluated in terms of 1-dimensional harmonic polylogarithms [6]. In [7, 8], the form factors for the axial vector, flavour singlet axial vector and flavour non-singlet axial vector currents were calculated using the same technique (see also [9]). Particular attention was payed to the prescription for the $D$-dimensional extension of the $\gamma_{5}$ Dirac matrix. A pragmatic approach was used: the diagrams not involving a closed triangular loop of fermions were evaluated performing the traces over the Dirac spinors with a naive anticommuting $\gamma_{5}$ [7], while the anomalous diagrams were evaluated in [8] with the prescription proposed in $[10,11]$. In all cases the corresponding Ward identities were checked and found to be fulfilled. In particular, the use of the prescription of $[10,11]$ in the evaluation of the anomalous diagrams in [8] breaks explicitly the anomalous Ward identities. These have to be restored, by performing a finite renormalization. The constant terms for the finite renormalization were calculated and their equality to the ones for the massless case, given in the $\overline{\mathrm{MS}}$ scheme in [11], was verified. Moreover, in order to check Ward identities, the pseudo-scalar form factor for the corresponding diagrams and the truncated matrix element of the gluonic operator $G \tilde{G}$ between the vacuum and an on-shell $Q \bar{Q}$ pair state were calculated in [8]. In [12], finally, the evaluation of the scalar and pseudo-scalar form factors was carried out in view of a completely differential description of the decay of a neutral Higgs boson, which can couple both to scalar and pseudo-scalar fermionic currents, into heavy quarks.

The calculation of the form factors is only a part of the complete determination of the heavy quark production matrix element. Nevertheless, it can also give very useful pieces of information on the precise determination of electroweak observables like, for instance, the forward-backward asymmetry $A_{F B}$ for heavy quarks at $e^{+} e^{-}$colliders [13]. Moreover, heavy quark form factors can give hints and restrictions in the search of new physics. If the mechanism of mass generation differs from the standard Higgs mechanism, deviations from the usual SM couplings of heavy particles ( $b$ or $t$ quarks) to photons and Z-bosons could be found. In [14], the NNLO QCD corrections to the anomalous magnetic moment and the weak axial-vector charge of $b$ and $t$ quarks were analyzed. It was found, in particular, that the upper bound on the $b$ quark magnetic moment coming from LEP1 data is saturated by the corrections due to two-loop perturbative QCD, which leave a limited room for new physics contributions to this quantity. Finally, the form factors give the possibility 
to calculate the two-loop QCD corrections to the cross section of $e^{+} e^{-} \rightarrow \gamma^{*}, Z^{*} \rightarrow t \bar{t}$ near the production threshold (c.f. [15] and refs. therein). This physical observable is important, for instance, for the precise determination of the top quark mass that enters in the determination of the constraints given by the SM on the Higgs mass. Although QCD perturbative calculations do not allow an investigation of the cross section directly at threshold because of the presence of Coulomb divergences, we can get precise information in the region $\alpha_{S} \ll \beta \ll 1, \beta=\sqrt{1-4 m^{2} / s}$ being the relative velocity of the quarks. Moreover, QCD perturbative calculations are important to extract the matching coefficients in the NRQCD perturbative series in powers of $\alpha_{S}$ and $\beta$.

Here we present for brevity only the cross section to $O\left(\alpha_{S}^{2}\right)$ at the production threshold of the $Q \bar{Q}$ pair, due to $\gamma$ and $Z$ boson exchange. Omitting in the threshold expansion terms of $O(\beta)$ (modulo the term $\sigma^{(0, V e)}$ in Eq. (1)) the $Q \bar{Q}$ contribution to the cross section is infrared finite by itself. Then we get, at NNLO, the following analytic result:

$$
\sigma=\sigma^{(0, V e)}\left\{1+\Delta^{(0, A x)}+C_{F} \frac{\alpha_{S}}{\pi} \Delta^{(1, V e)}\left(1+\Delta^{(0, A x)}\right)+C_{F}\left(\frac{\alpha_{S}}{\pi}\right)^{2}\left[\Delta^{(2, V e)}\left(1+\Delta^{(0, A x)}\right)+\Delta^{(2, A x)}\right]\right\},
$$

where the terms $\sigma^{(0, V e)}, \Delta^{(1, V e)}$, and $\Delta^{(2, V e)}$ are the contributions of the photon exchange to the treelevel, one-loop and two-loop cross section respectively (their analytic expressions to $O\left(\alpha_{S}^{2} \beta^{0}\right)$ can be found in [16]). $\Delta^{(0, A x)}$ and $\Delta^{(2, A x)}$ are the contributions, at the same order in $\alpha_{S}$ and $\beta$, of the $Z$-boson exchange and the $Z-\gamma$ interference, normalized to $\sigma^{(0, V e)}$ :

$$
\begin{aligned}
\Delta^{(0, A x)}= & \frac{8 m^{2} v_{Q}}{e_{Q} s_{W}^{2} c_{W}^{2}\left(4 m^{2}-m_{Z}^{2}\right)}\left[-v_{e}+\frac{2 m^{2}\left(v_{e}^{2}+a_{e}^{2}\right) v_{Q}}{e_{Q} s_{W}^{2} c_{W}^{2}\left(4 m^{2}-m_{Z}^{2}\right)}\right]-\beta^{2} \frac{4 m^{2}}{3 e_{Q} s_{W}^{2} c_{W}^{2}\left(4 m^{2}-m_{Z}^{2}\right)}\left\{6 v_{e} v_{Q}\right. \\
& \left.-\frac{m^{2}}{\left(4 m^{2}-m_{Z}^{2}\right)}\left[24 v_{e} v_{Q}+\frac{8\left(v_{e}^{2}+a_{e}^{2}\right)}{e_{Q} s_{W}^{2} c_{W}^{2}}\left(3 v_{Q}^{2}+a_{Q}^{2}-\frac{12 m^{2} v_{Q}^{2}}{\left(4 m^{2}-m_{Z}^{2}\right)}\right)\right]\right\} \\
\Delta^{(2, A x)}= & \frac{16 \zeta(2) m^{4} a_{Q}^{2}\left(v_{e}^{2}+a_{e}^{2}\right)}{e_{Q}^{2} s_{W}^{4} c_{W}^{4}\left(4 m^{2}-m_{Z}^{2}\right)^{2}} C_{F} .
\end{aligned}
$$

In Eqs. (2), (3) $m$ and $m_{Z}$ are the mass of the heavy quark and of the Z-boson respectively, $s_{W}$ $\left(c_{W}\right)$ the sine (cosine) of the weak mixing angle, $e_{Q}$ the charge of the heavy quark in units of the positron charge and $v_{f}=\frac{1}{2}\left(I_{f}^{(3)}-2 e_{f} s_{W}^{2}\right), a_{f}=\frac{1}{2} I_{f}^{(3)}$, with $f=e, Q$. The renormalization scale is taken equal to the heavy quark mass, $\mu=m$. These expressions are obtained expanding the exact result for the virtual cross section in powers of $\beta$, retaining only terms of $O\left(\beta^{0}\right)$. The diagrams that contribute to Eqs. (2), (3) are the ones of [1,7]. It turns out, in fact, that at $O\left(\beta^{0}\right)$, the anomalous diagrams do not play any role. The axial-vector contribution at $O\left(\alpha_{S}^{2}\right)$, which can be obtained to all orders in $\beta$ from the results of [7], is a new result. Here we give the leading term, i.e., the term proportional to $a_{Q}^{2}$ in Eq. (3) and in the $O\left(\beta^{2}\right)$ of Eq. (2). Notice that this term is of $O\left(\beta^{0}\right)$, while the axial-vector contribution at $O\left(\alpha_{S}\right)$ is of $O(\beta)$. The term $\Delta^{(2, A x)}$ is small compared to $\Delta^{(2, V e)}$. Nevertheless, at a high luminosity linear collider with polarized $e^{-}$and $e^{+}$beams one may eventually be able to disentangle the vector and axial-vector induced contributions to the $t \bar{t}$ cross section. (For a calculation of axial vector contributions in the context of Lippmann-Schwinger equations, see the 2 nd reference of [9].)

In conclusion we have computed the vector, axial-vector, scalar and pseudo-scalar vertices involving a pair of heavy quarks, $Q \bar{Q}$, to $O\left(\alpha_{S}^{2}\right)$, for arbitrary momentum transfer and mass of the heavy quarks. These form factors have a number of applications, including anomalous couplings, 
the $e^{+} e^{-} \rightarrow Q \bar{Q}$ cross section, the forward-backward asymmetry of heavy quarks, and the differential description of Higgs boson decays into $Q \bar{Q}$ pairs. Here we have briefly discussed the second order axial vector contributions to the $Q \bar{Q}$ threshold cross section, which is a new result. Although small these contributions may eventually be extracted from the $t \bar{t}$ threshold cross section measured at a high luminosity linear collider with polarized beams.

\section{References}

[1] W. Bernreuther, R. Bonciani, T. Gehrmann, R. Heinesch, T. Leineweber, P. Mastrolia and E. Remiddi, Nucl. Phys. B 706, 245 (2005).

[2] R. Barbieri, J. A. Mignaco and E. Remiddi, Nuovo Cim. 11A (1972) 824; Nuovo Cim. 11A (1972) 865. P. Mastrolia and E. Remiddi, Nucl. Phys. B 664 (2003) 341. R. Bonciani, P. Mastrolia and E. Remiddi, Nucl. Phys. B 676 (2004) 399. A.H. Hoang, M. Jezabek, J.H. Kühn and T. Teubner, Phys. Lett. B 338 (1994) 330. A.H. Hoang, J.H. Kühn and T. Teubner, Nucl. Phys. B 452 (1995) 173.

[3] S. Laporta and E. Remiddi, Phys. Lett. B 379 (1996) 283. S. Laporta, Int. J. Mod. Phys. A 15 (2000) 5087. C. Anastasiou and A. Lazopoulos, JHEP 0407 (2004) 046. F. V. Tkachov, Phys. Lett. B 100 (1981) 65. G. Chetyrkin and F. V. Tkachov, Nucl. Phys. B 192 (1981) 159. T. Gehrmann and E. Remiddi, Nucl. Phys. B 580 (2000) 485.

[4] A. V. Kotikov, Phys. Lett. B 254 (1991) 158; Phys. Lett. B 259 (1991) 314; Phys. Lett. B 267 (1991) 123. E. Remiddi, Nuovo Cim. A 110 (1997) 1435.

[5] R. Bonciani, P. Mastrolia and E. Remiddi, Nucl. Phys. B 661, 289 (2003) [Erratum-ibid. B 702, 359 (2004)]. R. Bonciani, P. Mastrolia and E. Remiddi, Nucl. Phys. B 690, 138 (2004). M. Czakon, J. Gluza and T. Riemann, Nucl. Phys. Proc. Suppl. 135 (2004) 83; Phys. Rev. D 71 (2005) 073009.

[6] A.B.Goncharov, Math. Res. Lett. 5 (1998), 497-516. D. J. Broadhurst, Eur. Phys. J. C 8 (1999) 311. E. Remiddi and J. A. M. Vermaseren, Int. J. Mod. Phys. A 15 (2000) 725. T. Gehrmann and E. Remiddi, Comput. Phys. Commun. 141 (2001) 296. D. Maître, arXiv:hep-ph/0507152.

[7] W. Bernreuther, R. Bonciani, T. Gehrmann, R. Heinesch, T. Leineweber, P. Mastrolia and E. Remiddi, Nucl. Phys. B 712, 229 (2005).

[8] W. Bernreuther, R. Bonciani, T. Gehrmann, R. Heinesch, T. Leineweber and E. Remiddi, Nucl. Phys. B 723, 91 (2005).

[9] J. Jersak, E. Laermann and P. M. Zerwas, Phys. Rev. D 25 (1982) 1218 [Erratum-ibid. D 36 (1987) 310]. J. H. Kühn and T. Teubner, Eur. Phys. J. C 9 (1999) 221. B. A. Kniehl and J. H. Kühn, Nucl. Phys. B329 (1990) 547.

[10] G. 't Hooft and M. J. G. Veltman, Nucl. Phys. B44 (1972) 189.

[11] S. A. Larin, Phys. Lett. B303 (1993) 113.

[12] W. Bernreuther, R. Bonciani, T. Gehrmann, R. Heinesch, P. Mastrolia and E. Remiddi, Phys. Rev. D 72 (2005) 096002.

[13] S. Catani and M. H. Seymour, JHEP 9907 (1999) 023. W. Bernreuther, R. Bonciani, T. Gehrmann, R. Heinesch, T. Leineweber, P. Mastrolia and E. Remiddi, in preparation.

[14] W. Bernreuther, R. Bonciani, T. Gehrmann, R. Heinesch, T. Leineweber, P. Mastrolia and E. Remiddi, Phys. Rev. Lett. 95 (2005) 261802.

[15] A.H. Hoang et al., Eur. Phys. J. directC 2 (2000) 1.

[16] A. Czarnecki and K. Melnikov, Phys. Rev. Lett. 80 (1998) 2531. M. Beneke, A. Signer and V.A. Smirnov, Phys. Rev. Lett. 80 (1998) 2535. 